\title{
Difference in Gender and Childbirth Costs and Their Association With Postpartum Depression
}

\author{
Farnaz Sadat Seyyed Ahmadinejad $^{1(}$, Naeimeh Tayebi $^{{ }^{*}(\mathbb{C}}$, Mehrdad Khatami $^{{ }^{(}}$
}

\begin{abstract}
Objectives: Pregnancy and childbirth are physiological events and they are pleasant in most cases although they are occasionally associated with complications that can be dangerous for the mother and the infant if left unattended. The child's gender can have emotional-psychological, economic, social, and normative dimensions for different people. Therefore, the present study was done to investigate the difference in gender and the costs of childbirth and evaluate their association with postpartum depression.

Materials and Methods: The present cross-sectional study included 260 primiparous women as the sample who were selected by the convenient-nonprobability sampling method. Data collection tools included the Edinburgh Postnatal Depression Questionnaire and forms related to demographic information, infant gender, and the like, which were completed through interviews.

Results: In this study, 260 women were evaluated after delivery, and the prevalence of postpartum depression was $56.9 \%$. There was a statistically significant relationship between postpartum depression and unwanted pregnancy, delivery method, weeks of pregnancy, social and economic costs of having a child, and gender. However, no significant relationship was found between postpartum depression and maternal aging, and the individual costs of having a child.

Conclusions: Postpartum depression has a high prevalence in our society in which almost one mother out of two mothers is involved with depression problems, which can affect the health of the mother, her infant, and the whole family. Therefore, it seems that postpartum depression should be considered as an essential component of postpartum care.

Keywords: Gender differences, Child, Postpartum depression, Women, Population growth
\end{abstract}

\section{Introduction}

Pregnancy and childbirth are considered as major evolutionary trends for most women. Physical, intrapersonal, and family compatibilities are necessary for the successful adaptation of pregnancy and childbirth. The stresses experienced by an inexperienced mother are extremely much and include disturbances in normal activities (1). Although pregnancy and childbirth are physiological events and pleasant in most cases, they are occasionally accompanied with complications that can be dangerous to the mother and the infant if they are ignored $(1,2)$. Pregnancy triggers a wide variety of emotions in women, ranging from excitement and enthusiasm to anxiety, stress, and depression. Some women experience dramatic fluctuations in their emotions. Previous evidence shows that women who suffer pregnancy complications are more likely to demonstrate depression compared to women with normal pregnancies (3).

Postpartum depression is the most common mental health problem among women affecting 10-15\% of mothers worldwide $(4,5)$. Postpartum depression is a disorder that occurs in some women between two and six weeks after showing different symptoms such as crying, disappointment, insecure mood, feeling inadequate, loss of appetite, feeling guilty, suicidal thoughts, sleep disturbances, decreased concentration and memory, fatigue, irritability, inability to play a role as a mother, and a feeling of inadequacy in taking care of the infant $(6,7)$.

Severe postpartum hormonal changes involve severe mental consequences that range from mild to severe depression afterbirth. This problem can continue if it is not treated after delivery (8). According to the American Psychiatric Association, postpartum depression includes periods of depression occurring during pregnancy or 4 weeks afterbirth (9).

Many mothers develop mood disturbances in the first days of childbirth. The mild type of this state with sadness or crying is called postpartum blues. The peak of postpartum blues is up to 5 days after childbirth and then subsides gradually (6). The causes of postpartum blues are not well known. However, some of the possible causes include early lochia, fatigue due to the lack of sleep, fear of reduced attractiveness after childbirth, anxiety about the inability to care for the baby, and a history of depression in the period before delivery. The involvement of hormonal agents such as endorphin, estrogen, and progesterone levels after delivery has also been reported in this regard (9). The more severe type of this disorder, which is characterized by depressed mood, increased anxiety, and insomnia, is called postpartum depression. 
Key Messages

- The child's gender can have emotional-psychological, economic, social, and normative dimensions for different people.

- Psychological interventions have been effective in reducing depression and can improve the quality of a mother's life without having any adverse effects or the side effects of medication.

- the relationship between unwanted pregnancy and depression suggests the need for an increase in the quality of family planning programs, particularly the training on the proper use of contraceptives.

The prevalence of this disorder in other countries is about $30 \%(10,11)$, and it is reported to be $20 \%-42 \%$ in Iranian studies (12).

Postpartum depression is a multi-agent disorder. Researchers have introduced two psychosocial and social models in the development of this disorder (13). The cause of postpartum depression is not definitely known, but hormonal changes occurring after birth are likely to be related to this disorder. The level of estrogen, progesterone, and other hormones are extremely reduced from the birth of the child although there is no significant diversity in the hormonal difference between women who experience postpartum depression and other women who do not experience this disorder. However, some women may be more vulnerable to these hormonal changes compared to other women.

The child's gender can have emotional-psychological, economic, social, and normative dimensions for different people. For example, several benefits of child gender include having someone that parents can love and care, someone who helps the household economy, helps at home and ensures the safety of aging, continues the generation, and promotes the status of parents among close relatives $(14,15)$.

In terms of etiology, there are multiple theories for the recognition of postpartum depression that include biologic factors, increased cortisol urinary excretion, neurotransmitters and genetic theories, social factors such as social support, life tensions, cultural factors governing the society, and the level of readiness for motherhood. Other reasons for this disorder are the need for psychological adaptation after birth, the lack of sleep, and taking care of the infant. Accepting the 24-hour responsibility of caring for a newborn infant requires a major mental and lifestyle adaptation in most mothers $(16,17)$. These physical and psychological stresses are usually accompanied by the mother's inadequate rest. Moreover, stress, the lack of sleep, undesirable nutrition, the lack of mother support from a spouse, and a history of previous mental disorders in the mother are factors aggravating the disease.
Considering that the postpartum period can be highly tense that provoke psychological illnesses and by considering the cultural and social issues affecting societies in the occurrence of depression, it can be found that whether the importance of gender differences and child birth costs is involved in the incidence of postpartum depression. Accordingly, it will be possible to educate childcare skills to reduce the incidence of this disorder which, leads to more indirect costs due to disturbances in maternal, child, and family relationships in addition to direct costs for the health care system. Given that the results of previous studies are inconsistent with the demographic variables affecting postpartum depression, it seems that more evidence is needed to address this important health problem and the factors affecting it in further studies, which are necessary in this regard. Therefore, the present study was done to determine the difference between gender and postpartum depression in women who referred to healthcare centers.

\section{Materials and Methods}

This cross-sectional, analytical, and epidemiological study was done in 2015-2016. This study is derived from a research project with the code number 36.95 and the ethics code of MUBAM.REC.1395.2. The population of this study included all primiparous women who referred to healthcare centers in Bam. Considering that no similar study was found during the extensive search of data resources to show an approximate estimate of the child's ratio and postpartum depression, the sample size was selected based on the results of the guideline study on 30 subjects. Using the formula for estimating the relationship with a confidence interval of $95 \%$ and a test power of $80 \%$, the estimated sample size was approximately 260 people.

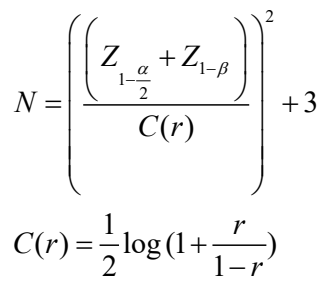

The study inclusion criteria were Iranian women living in Bam, being in the age range of 16-35 years old, being primiparous, having singleton infants, and being in the 8th week after childbirth (due to the referral of mothers for their two-month-old-baby vaccination and better access to all mothers within a specific postpartum period, all specimens were screened for 8 weeks postpartum). Other criteria were body mass index (BMI) less than $26 \mathrm{~kg} . \mathrm{m}^{2}$, no use of medicine, no chronic illness ${ }^{[1]}$, no abortion history, lack of a history of infertility and high-risk pregnancy ${ }^{[2]}$, no history of depression before and during pregnancy, and non-occurrence of life-threatening events ${ }^{[3]}$ over the past six months. On the other hand, the exclusion criteria were the occurrence of crisis or stressful incidence, women's 
desire to leave the study, preterm delivery, sick neonate, an infant weighing less than $2500 \mathrm{~g}$, a history of emergency cesarean section, and hard baby delivery.

In the first step, a cluster quota sampling method was used to select samples. Thus, first, a list of all health centers in Bam was prepared and then three health centers were randomly selected among them. Cluster sampling saves time and cost and avoids the dispersal of the selected samples in the city. Then, two days were considered for sampling from each center by drawing lots from the days of the week. Next, eligible women were included by the simple non-probabilistic sampling method after explaining the goals and method of the study and obtaining written informed consent. The present study was done after obtaining the permission of the deputy research director and the confirmation of the Ethics Committee of Bam University of Medical Sciences, coordinating with the city health center, getting a referral from the security center, presenting it to the authorities of health-care centers, and making coordination for the time of distribution of the study tools.

Women eligible for the study inclusion referring to the health center for the vaccination of their two-monthold child completed their informed consent before participating in the vaccination. Therefore, the researcher asked the study subjects to complete the Individual and Midwifery Information Questionnaire, Questionnaire of Value of Children, and Edinburgh Postnatal Depression Questionnaire after examining the study inclusion and exclusion criteria and representing the goals of the study (18). Individual and Midwifery Information Questionnaire included questions about individual characteristics and women's and midwifery information in addition to disease records of the research that were completed by the research unit.

The questionnaire included two parts of the benefits and costs of having a child (18). The part related to the costs of having a child contained 217 items and measured by a 5 -point Likert-type scale of "not at all important" to "very important" ranging from 1 to 5 . The obtained results were categorized into 3 (5 items) economic, social, and individual dimensions. The obtained score in each dimension was determined based on the mean value, and higher scores indicated the higher costs of having a child in the relevant dimension.

Edinburgh Postnatal Depression Questionnaire encompassed 10 multiple choice questions on a Likerttype scale, and each question had a score of $0-3$. This questionnaire was confirmed for the evaluation of postpartum depression. Score 10 or more in this questionnaire showed postpartum depression and a score of less than 10 indicated a normal person in terms of postpartum depression. The average length of time for answering questions was 15 minutes.

In addition, women were ensured of data confidentiality. Data were collected using SPSS software (version 16), and descriptive and inferential statistics were used to analyze the data. Descriptive statistics including central and dispersion indicators such as the mean, standard deviation, frequency distribution, and charts were used to describe the characteristics of research units in each group. Further, the natural distribution of quantitative variables was determined by using the Kolmogorov-Smirnov test. Mann-Whitney test or $t$ test was used to investigate quantitative variables if necessary. Finally, the $\chi^{2}$ test was and non-parametric tests were applied for qualitative and sequential quality variables, respectively. A $P$ value of less than 0.05 was considered statistically significant.

\section{Results}

This study evaluated 260 women with an age range of 1635 years old and a mean age of 25 . Among the studied women, 195 (75\%) cases were housewives and only 39 (15\%) of them were employees. Other 12 (5\%) women were university students, 8 (3\%) cases had home jobs, and 6 (75\%) cases were self-employed. In terms of housing status investigations, 101 (39\%) women were the owners of a house and 159 (61\%) cases had leased houses. The average number of female children in the studied population was $102(39 \%)$, and $158(61 \%)$ cases were males.

Out of 260 subjects, 39 (15\%) and $39(15 \%)$ cases were economically ineffective, and $215(84 \%)$ of them were at the expense and 3 (1\%) were more than adolescents. In terms of the housing status survey, the housing situation was occupied by 101 (39\%) and 159 (61\%) people. The average number of female children was 102 (39\%) and 158 (61\%).

Table 1 presents the coefficients of the regression model between postpartum depression and the dimensions of the cost of having a child. As shown, the social and economic dimensions of the cost of having a child are significant at the level of 0.05 , and these dimensions perfectly predict postpartum depression although the individual dimension of the cost of having a child is not significant at level of 0.05 . In the statistical results of this study (Table 2), the economical dimension with a mean of $2.52 \pm 0.74$ had the

Table 1. Coefficients of the Regression Model Between Postpartum Depression and the Cost of Having a Child

\begin{tabular}{lccccc}
\hline Variable & Non-standard Coefficient & Standard Deviation & Standardized Coefficient & T-test Statistics & $\boldsymbol{P}$ Value \\
\hline Width from origin & -0.867 & 0.327 & - & -2.649 \\
Economic costs & -0.253 & 0.032 & -0.367 & -7.903 \\
Social costs & 0.847 & 0.054 & 0.994 & 15.771 \\
Individual costs & -0.017 & 0.057 & -0.021 & -0.303 \\
\hline
\end{tabular}


Table 2. Comparison of the Average Cost of Having a Child and its Dimensions With the Father's Job in the Studied Women

\begin{tabular}{|c|c|c|c|c|}
\hline Father's Job & Cost of Having a Child & Economical Cost & Social Cost & Individual Cost \\
\hline Unemployed & $2.79 \pm 0.45$ & $2.20 \pm 0.66$ & $2.41 \pm 0.99$ & $3.19 \pm 0.09$ \\
\hline Self-employed & $2.79 \pm 0.55$ & $2.48 \pm 0.73$ & $2.74 \pm 0.58$ & $2.91 \pm 0.61$ \\
\hline Worker & $2.75 \pm 0.60$ & $2.51 \pm 0.76$ & $2.71 \pm 0.59$ & $2.84 \pm 0.69$ \\
\hline Employee & $2.87 \pm 0.51$ & $2.65 \pm 0.74$ & $2.81 \pm 0.56$ & $2.97 \pm 0.58$ \\
\hline Test statistics & 10.383 & 25.922 & 27.269 & 16.998 \\
\hline$P$ value & 0.961 & $0.039^{*}$ & 0.343 & 0.882 \\
\hline
\end{tabular}

Note. *Significant at 0.05 .

Table 3. Comparison of the Average Cost of Having a Child and its Dimensions With the Mother's Job in the Studied Women

\begin{tabular}{|c|c|c|c|c|}
\hline Mother's Job & Cost of Having a Child & Economic Cost & Social Cost & Individual Cost \\
\hline Housewife & $2.81 \pm 0.54$ & $2.54 \pm 0.72$ & $2.77 \pm 0.58$ & $2.90 \pm 0.60$ \\
\hline University student & $2.87 \pm 0.56$ & $2.50 \pm 0.77$ & $2.59 \pm 0.94$ & $3.15 \pm 0.48$ \\
\hline Home job & $2.82 \pm 0.32$ & $2.04 \pm 0.82$ & $2.69 \pm 0.11$ & $3.12 \pm 0.35$ \\
\hline Self-employed & $2.71 \pm 0.57$ & $2.11 \pm 0.93$ & $2.57 \pm 0.43$ & $2.96 \pm 0.69$ \\
\hline Employee & $2.78 \pm 0.58$ & $2.58 \pm 0.76$ & $2.69 \pm 0.63$ & $2.90 \pm 0.66$ \\
\hline Test statistics & 17.829 & 25.725 & 35.184 & 10.904 \\
\hline$P$ value & 0.334 & $0.012^{*}$ & $0.019^{*}$ & 0.949 \\
\hline
\end{tabular}

Table 4. Comparison of the Average Cost of Having a Child and its Dimensions With the Housing Situation in the Studied Women

\begin{tabular}{lccc}
\hline Housing Situation & Having a Child & Economic Cost & Social Cost \\
\hline Owner of a house & $2.75 \pm 0.57$ & $2.50 \pm 0.77$ & $2.68 \pm 0.59$ \\
Leasing of a house & $2.84 \pm 0.52$ & $2.53 \pm 0.72$ & $2.79 \pm 0.60$ \\
Test statistic & 3.490 & 1.611 & 2.438 \\
$P$ value & 0.479 & 0.657 & 0.786 \\
\hline
\end{tabular}

Table 5. Comparison of the Average Cost of Having a Child and its Dimensions With the Economic Situation in the Studied Women

\begin{tabular}{lccc}
\hline The Economic Situation & Cost of Having a Child & Economic Cost & Social Cost \\
\hline A reasonable rate & $2.73 \pm 0.60$ & $2.41 \pm 0.76$ & $2.69 \pm 0.60$ \\
At a reasonable rate & $2.81 \pm 0.53$ & $2.52 \pm 0.73$ & $2.75 \pm 0.60$ \\
More than enough & $3.42 \pm 0.16$ & $3.33 \pm 0.57$ & $3.33 \pm 0.41$ \\
Test statistics & 7.540 & 7.524 & 7.315 \\
$P$-value & 0.480 & 0.275 & 0.695 \\
\hline
\end{tabular}

lowest dimension of the child value and the individual dimension with the mean of $3.92 \pm 0.6$ had the highest dimension of the cost of having a child.

Tables 2-5 provide the difference between the percentage of the costs of having a child and its dimensions with the mother's age, education level, parents' job, housing situation, economic status, and child's gender in the studied women. A significant relationship was observed between the father's job and the cost of the child $(P=$
$0.03)$, the mother's job and the economic and social costs of the child $(P=0.01)$, and the child's gender and the cost of having a child $(P=0.001)$. However, no significant relationship was found between maternal age $(P=0.69)$, mother's education $(P=0.83)$, housing situation $(P=$ $0.47)$, and economic status $(P=0.48)$. The Mann-Whitney test was used to examine the relationship between the two variables of the child's gender and the incidence of postpartum depression $(P=0.001)$.

Table 6. Comparison of the Percentage of Postpartum Depression, the Cost of Having a Child and its Dimensions With the Child's Gender in the Studied Women

\begin{tabular}{lcccc}
\hline Child's Gender & Postpartum Depression & Cost of Having a Child & Economic Cost & Social Cost \\
\hline Girl & $0.73 \pm 0.43$ & $2.45 \pm 0.40$ & $2.38 \pm 0.48$ & $2.35 \pm 0.51$ \\
Boy & $0.99 \pm 0.53$ & $3.03 \pm 0.50$ & $2.60 \pm 0.85$ & $3.00 \pm 0.49$ \\
Test statistics & 52.264 & 176.055 & 147.471 & 142.658 \\
$P$-value & $0.001^{*}$ & $0.001^{*}$ & $0.001^{*}$ & $0.001^{*}$ \\
\hline
\end{tabular}

*Significant at 0.05 . 
Table 6 summarizes the comparison of the percentage of postpartum depression, the cost of having a child, and its dimensions with the child's gender in the studied women. Finally, a significant relationship was observed between postpartum depression and the cost of having a boy $(P=$ 0.001).

\section{Discussion}

The present study was done to evaluate the structure of gender difference and the costs of having a child in Bam and to compare them among the women of different ages and its relationship with postpartum depression. Postpartum depression is one of the problems that threats the life of the mother, the infant, and the family. Often women who suffer from this disorder do not attempt to receive treatment, and therefore, receive no treatment. Consequently, they may remain depressed for more than one year after childbirth, which can have a negative effect on their relationship with the spouse, it may seriously affect the relationship between the mother and the child leading to cognitive and emotional delays in the child and even child negligence (19), and overall profound effects on the health and well-being of the child (20).

In the present study, the prevalence of depression was $56.9 \%$, which is much higher than what was expected. As it was observed in statistics, postpartum depression is more common in developing societies compared to developed societies (23). However, it seems that the issue of social-economic development alone cannot justify the high prevalence of this disorder in our study and other reasons (e.g., cultural issues and traditions in this case) probably have a role in conducting studies in this field that can help in accepting or rejecting the theory. In the review of data sources, Bina explains that different cultures have varied beliefs and traditions that can affect the prevalence of postpartum depression.

In the study of Inandi et al (21), there was a significant relationship between postpartum depression and wife's education, high postpartum delivery, unwanted pregnancy, and incompatibility with the spouse. However, no significant relationship was found between postpartum depression and the age difference of couples, the level of education and employment of women, history of abortion and stillbirth, the age and type of delivery, the history of depression in the past, BMI at the beginning of pregnancy, and infant gender. In their study, Pham and Hardie (22) demonstrated no significant relationship between infants' gender and postpartum depression after 8-12 weeks of postpartum investigation in Asian-American women. However, in another study examining postpartum depression, a significant relationship was reported between postpartum depression and maternal age, type of family, domestic violence by the spouse, the number of pregnancies, and the gender of the infant.

Alharbi and Abdulghani (23) reported anemia occurring in the postpartum period as the most frequent factor in this regard while other factors such as demographic factors, child gender, and delivery method had no significant relationship with postpartum depression. However, the female gender of the child and cesarean section increased the incidence of postpartum depression (24). Moreover, Lashkaripour et al (25) found a significant relationship between the occurrence of postpartum depression and the unwanted gender of the infant (16). Likewise, Xie et al suggested that postpartum depression was more prevalent among women who had a girl in comparison with those who had a boy (26). In the study of Bodnar et al (27), the female gender of the second child was a major contributor to the incidence and severity of postpartum depression (28). Conversely, Perfetti et al (29) found that women with a boy were more likely to suffer postpartum blues. In a study conducted in Western countries, there was no relationship between the gender of the infant and postpartum depression while, in Asian societies, a relationship was reported between postpartum depression and the infant's gender (26). The rate of postpartum depression is significantly higher for mothers who have a son compared to those who have a daughter. The cost of having a boy and its dimensions is more than a girl. This problem can be explained by considering the cultural context of the area and the early marriage of the girl and the role of the boy in helping the family or even the possibility of being rooted, in this issue, from the childhood, of a mother whose family had a more positive attitude toward the boy and considered the baby son as a sign of blessing in the family as a matter of concern to mothers for the future of their baby son and their higher cost (Table 6). In a study conducted in Zahedan (25), the prevalence of postpartum depression was $33 \%$ and factors such as the type of delivery, maternal age, gender preferences for children from parents, educational level, previous history of depression, previous history of referrals to psychiatrists, and the use of psychosocial drugs were associated with depression in the mother. In another study in Kerman (30), the prevalence of depression was reported to be $31 \%$ and the results showed that the first delivery, abortion history, child death, unwanted pregnancy, and gender preferences for children were associated with an increased prevalence of depression.

Mood disorders in pregnancy and the postpartum period are common and considered as a public health issue. In recent decades, researchers have paid great attention to these subjects and showed that mood illness related to childbirth can lead to serious complications for the mother, the fetus, the newborn, and the family $(31,32)$. Evidence indicates that postnatal depression is an extension of antenatal depression, which continues postpartum. This problem can have negative effects on infant development and can cause behavioral, cognitive, social, and emotional problems in the infant, which could continue throughout childhood (31-33). Postpartum depression in the event of a deterioration and persistence 
for more than 6 months will cause problems for children. In these situations, in addition to behavioral disorders, the GCSE Maths exam results in a low score at the age of 16, and at the age of 18 , they show symptoms of depression.

According to the findings of this study, there was a significant relationship between the wanted or unwanted pregnancy, marital problems, and postpartum depression; because unwanted pregnancy indicates that the mother is not ready to accept the child's responsibility. This issue can be a factor to increase the prevalence of postpartum depression. The results of the study by Wewerinke et al indicate this claim (34). Iranfar et al (35) also mentioned unwanted pregnancies as risk factors for postpartum depression. Marital problems also lead to the poor support of the husband for a pregnant and a childbearing woman. Horowitz et al (36) mentioned poor husband support as one of the major causes of postpartum depression $(37,38)$.

In an urban community, childlessness is not acceptable at all because the psychological life and the continuity of the family depend on the existence of the child. The social motivation for childbearing is highly strong and the child's desirability is related to the number of them to the psychological and economic costs and child-raising limitations. Urban women think about these costs, and in many cases, the quantity of children is subject to the individual's perceptions of these costs, which is why the large family has little utility. Urban changes and urbanindustrial constraints such as housing shortages and high living costs affect the mental efficiency of children, and economic costs play a major role in psychological values. In Rajabi and Hasheminia's study (39), one or two children are referred to as the child's desirable number. Because of the growing number of children, economic constraints arise. The cost of having a child and its dimensions in a boy's son is more than a girl that is consistent with the current study.

Due to the lack of proper cooperation of some women in answering the questionnaire because of some physical problems or distractions, the inquirer gained the trust of women by creating a good relationship before conducting an interview. Distributing the questionnaire by the inquirer and completing all the items by the participants were the other problems. To reduce these restrictions, while releasing the units to study and exit, they were assured of data confidentiality and that the results would be reported in general. In addition, the researchers tried to get their cooperation by explaining the goals of the research, and the questionnaire was completed by the research units at the waiting time for receiving care in health care centers.

These programmers need to take into account the complex and multi-factorial nature of psychological problems associated with pregnancies. Spouses, family members, and health professionals need to invest the greatest efforts in such situations. Mothers need to receive maximum support, care, and encouragement as the health of their newborn largely relies on their own health.
Strengths and Limitations of the study

The strengths of this study included the probabilistic and multi-stage sampling method of the study design, the division of the number of samples based on the target society in Bam, and the equal chance of selecting women. In addition, the sampling of healthy women (based on their health records or their self-declaration) was performed on women who referred to healthcare centers. This greatly improved the generalizability of the results while minimizing the probability of bias. The importance of this issue in the policymaking of the country and the lack of similar studies across the country are the strength points of this study. One of the limitations of this study was its cross-sectional nature. Therefore, some future studies may be required to measure some variables. In addition, the difference in the understanding of research units from the raised questions can be mentioned as another limitation of this study. Sampling was conducted only in the urban areas of Bam due to the problems and transportation costs of sampling while not including rural areas. Thus, it is suggested that future studies be carried both in the rural and urban areas simultaneously. The results of this study are expected to provide sub-structural data on one of the factors affecting women in terms of postpartum depression. In addition, these results can be useful for training regarding reducing the sensitivity to child gender and training parenting skills.

\section{Conclusions}

Postpartum depression has a high prevalence in our society in which almost one mother out of two mothers is involved with a depression problem that can affect the health of the mother, infant, and the whole family.

Based on the results, the rate of postpartum depression was significantly higher for mothers who had a son compared to those who had a daughter. The cost of having a child and its dimensions for a boy was more than a girl. Accordingly, the cultural context of the area and the early marriage of the girl's family and the influence of the boy in helping the family, or even the possibility of being rooted in this issue should be considered in explaining this problem. Further, it should be mentioned that the childhood of the mother whose families had a more positive attitude toward a son and considered the son's birth as a sign of blessing in the family as a matter of concern to mothers for the future of their son's child and their higher cost can be highly effective.

Given that early interventions, careful follow-ups, and treatments are associated with the best prognosis, it seems that screening for postpartum depression and the treatment of depressed mothers should be considered as an essential component of postpartum care. In addition, the relationship between unwanted pregnancy and depression suggests the need for an increase in the quality of family planning counseling programs, particularly the training on the proper use of contraceptives for reducing 
unwanted pregnancies. Special attention should be paid to the mothers who had an unwanted pregnancy and those with a history of depression during pregnancy and postpartum.

Psychological interventions have been effective in reducing depression and can improve the quality of a mother's life without having any adverse effects or the side effects of medication. Postpartum counseling sessions affect the physical, emotional, and psychological well-being of families. Attending the counseling sessions can increase parental satisfaction, self-esteem, and the interaction of family members with each other. Regarding the high prevalence of postpartum depression and considering that medication during postpartum and breastfeeding can have significant effects on the mother and infant, using therapies and psychological interventions such as problem-solving skills and relaxation is considered necessary. Regarding the relatively high prevalence of postpartum depression and its relationship with stressful events, it is recommended to prevent this disorder by identifying the sources of stress, coping skills training, and activating supportive systems (e.g., family, friends, and the like) in order to take a step, even a small one, toward the health of mothers during the pregnancy period. Further recommended could be as follows:

- Informing women about the symptoms of high-risk pregnancy and its consequences, and asking them to refer to a health professional;

- Informing women about how to prevent an unwanted pregnancy;

- Helping couples improve their relationship during and after pregnancy;

- Helping families keep a balanced attitude toward the child's gender.

\section{Authors' Contribution}

NT and FS: concept and design. NA and MK: data collection and interpretation of the data. NT and FS: performing of the study and writing of the draft. All authors read and approved the study.

\section{Conflict of Interests}

Authors declare that they have no conflict of interests.

\section{Ethical Issues}

This research was approved by the Ethics and Human Subjects Committee of Bam University of Medical Sciences with the ethics code of MUBAM.REC.1395.2.

\section{Financial Support}

This research was supported by Bam University of Medical Sciences.

\section{Acknowledgments}

The authors sincerely thank all staff of the Pastor Hospital for their help.

\section{Endnotes}

[1] Underlying diseases include diabetes, cardiovascular disease, hypertension, epilepsy, migraine, thyroid disease, connective tissue disease, asthma, kidney disease, and anemia.

[2] Hypertension in pregnancy and preeclampsia, eclampsia, bleeding, premature uterine contractions, fetal abnormalities, intrauterine growth restriction, placental abruption, and placenta previa.

[3] Death of a family member, family member illness, financial problems, accidents, firing or losing the job of samples, or their spouse, family disputes

\section{References}

1. Tol WA, Purgato M, Bass JK, Galappatti A, Eaton W. Mental health and psychosocial support in humanitarian settings: a public mental health perspective. Epidemiol Psychiatr Sci. 2015;24(6):484-494. doi:10.1017/s2045796015000827

2. Kingston D, Tough S. Prenatal and postnatal maternal mental health and school-age child development: a systematic review. Matern Child Health J. 2014;18(7):1728-1741. doi:10.1007/ s10995-013-1418-3

3. O'Hara MW, Swain AM. Rates and risk of postpartum depression—a meta-analysis. Int Rev Psychiatry. 1996;8(1):3754. doi:10.3109/09540269609037816

4. Veisani Y, Delpisheh A, Sayehmiri K, Rezaeian S. Trends of postpartum depression in Iran: a systematic review and meta-analysis. Depress Res Treat. 2013;2013:291029. doi:10.1155/2013/291029

5. Kamalifard M, Bayati Payan S, Panahi S, Hasanpoor S, Babapour Kheiroddin J. Paternal postpartum depression and its relationship with maternal postpartum depression. J Holist Nurs Midwifery. 2018;28(2):115-120. doi:10.29252/ hnmj.28.2.115

6. Giallo R, Wade C, Cooklin A, Rose N. Assessment of maternal fatigue and depression in the postpartum period: support for two separate constructs. J Reprod Infant Psychol. 2011;29(1):69-80. doi:10.1080/02646838.2010.513050

7. Misra DP, Guyer B, Allston A. Integrated perinatal health framework. A multiple determinants model with a life span approach. Am J Prev Med. 2003;25(1):65-75. doi:10.1016/ s0749-3797(03)00090-4

8. Gavin $\mathrm{NI}$, Meltzer-Brody S, Glover V, Gaynes BN. Is population-based identification of perinatal depression and anxiety desirable? a public health perspective on the perinatal depression care continuum. In: Milgrom J, Gemmill AW, eds. Identifying Perinatal Depression and Anxiety: EvidenceBased Practice in Screening, Psychosocial Assessment, and Management. Wiley-Blackwell; 2015:11-31.

9. Seimyr L, Welles-Nyström B, Nissen E. A history of mental health problems may predict maternal distress in women postpartum. Midwifery. 2013;29(2):122-131. doi:10.1016/j. midw.2011.11.013

10. Sadock BJ, Sadock VA. Kaplan and Sadock's Synopsis of Psychiatry: Behavioral Sciences/Clinical Psychiatry. Lippincott Williams \& Wilkins; 2011.

11. Wee KY, Skouteris H, Pier C, Richardson B, Milgrom J. Correlates of ante- and postnatal depression in fathers: a systematic review. J Affect Disord. 2011;130(3):358-377. doi:10.1016/j.jad.2010.06.019

12. Rafiee B, Akbarzade M, Asadi N, Zare N. Comparison of attachment and relaxation training effects on anxiety in third trimester and postpartum depression among primipara women. Hayat. 2013;19(1):76-88. [Persian].

13. Collins NL, Dunkel-Schetter C, Lobel M, Scrimshaw SC. Social support in pregnancy: psychosocial correlates of birth outcomes and postpartum depression. J Pers Soc Psychol. 1993;65(6):1243-1258. doi:10.1037//0022-3514.65.6.1243

14. Cousineau TM, Domar AD. Psychological impact of infertility. Best Pract Res Clin Obstet Gynaecol. 2007;21(2):293-308. doi:10.1016/j.bpobgyn.2006.12.003

15. Hoffman LW, Hoffman ML. The Value of Children to Parents. Ann Arbor: Department of Population Planning School of 
Public Health, University of Michigan; 1973.

16. Albert I, Trommsdorff G, Mayer B, Schwarz B. Value of children in urban and rural Indonesia: socio-demographic indicators, cultural aspects, and empirical findings. In: Trommsdorff G, Nauck B, eds. The Value of Children in Cross-Cultural Perspective. Pabst Science Publishers; 2005:171-207.

17. Qvortrup J. Sociology: societal structure, development of childhood, and the well-being of children. In: Ben-Arieh A, Casas F, Frønes I, Korbin J, eds. Handbook of Child WellBeing. Dordrecht: Springer; 2014:663-707. doi:10.1007/97890-481-9063-8_138

18. Fazeli E, Golmakani N, Taghipour A, Shakeri MT. Intergenerational comparison of value of children among urban women population in Mashhad, Iran, 2014. J Midwifery Reproductive Health. 2016;4(4):732-740. doi:10.22038/ jmrh.2016.7306

19. Ntozi JP. The desire for children in tropical Africa: the influence of relatives on fertility decisions in Ankole, Uganda. J Biosoc Sci. 1990;22(4):433-446. doi:10.1017/s0021932000018848

20. Siegenthaler AL, Bigner JJ. The value of children to lesbian and non-lesbian mothers. J Homosex. 2000;39(2):73-91. doi:10.1300/J082v39n02_04

21. Inandi T, Elci OC, Ozturk A, Egri M, Polat A, Sahin TK. Risk factors for depression in postnatal first year, in eastern Turkey. Int J Epidemiol. 2002;31(6):1201-1207. doi:10.1093/ ije/31.6.1201

22. Pham A, Hardie T. Does a first-born female child bring mood risks to new Asian American mothers? J Obstet Gynecol Neonatal Nurs. 2013;42(4):471-476. doi:10.1111/15526909.12226

23. Alharbi AA, Abdulghani HM. Risk factors associated with postpartum depression in the Saudi population. Neuropsychiatr Dis Treat. 2014;10:311-316. doi:10.2147/ndt.s57556

24. Golmakani N, Fazeli E, Taghipour A, Shakeri MT. Relationship between gender role attitude and fertility rate in women referring to health centers in Mashhad in 2013. Iran J Nurs Midwifery Res. 2015;20(2):269-274.

25. Lashkaripour K, Bakhshani NM, Hokmabadi S, Sajjadi SAR, Safarzadeh Sarasiyabi A. Postpartum depression and related factors: a 4.5 months study [Persian]. Journal of Fundamentals of Mental Health. 2011;13(4):404-412. doi:10.22038/ jfmh.2011.928

26. Xie RH, He G, Liu A, Bradwejn J, Walker M, Wen SW. Fetal gender and postpartum depression in a cohort of Chinese women. Soc Sci Med 2007;65:680-4.
27. Bodnar LM, Wisner KL. Nutrition and depression: implications for improving mental health among childbearing-aged women. Biol Psychiatry. 2005;58(9):679-685. doi:10.1016/j. biopsych.2005.05.009

28. Henz U. Gender roles and values of children: childless couples in East and West Germany. Demogr Res. 2008;19:1451-1500. doi:10.4054/DemRes.2008.19.39

29. Perfetti J, Clark R, Fillmore CM. Postpartum depression: identification, screening, and treatment. WMJ. 2004;103(6):5663.

30. Ghaffarinejad AR, Khobyari F, Pouya F. Prevalence of postpartum depression in Kerman. Iran J Psychiatry Clin Psychol. 1999;5(1):24-30. [Persian].

31. Walker LO, Sterling BS, Guy S, Mahometa MJ. Cumulative poor psychosocial and behavioral health among low-income women at 6 weeks postpartum. Nurs Res. 2013;62(4):233242. doi:10.1097/NNR.0b013e31829499ac

32. Deng AW, Xiong RB, Jiang TT, Luo YP, Chen WZ. Prevalence and risk factors of postpartum depression in a populationbased sample of women in Tangxia Community, Guangzhou. Asian Pac J Trop Med. 2014;7(3):244-249. doi:10.1016/s19957645(14)60030-4

33. Easterbrooks MA, Bartlett JD, Raskin M, et al. Limiting home visiting effects: maternal depression as a moderator of child maltreatment. Pediatrics. 2013;132 Suppl 2:S126-133. doi:10.1542/peds.2013-1021K

34. Wewerinke A, Honig A, Heres MH, Wennink JM. [Psychiatric disorders in pregnant and puerperal women]. Ned Tijdschr Geneeskd. 2006;150(6):294-298.

35. Iranfar S, Shakeri J, Ranjbar M, Nazhadjafar P, Razaie M. Is unintended pregnancy a risk factor for depression in Iranian women? East Mediterr Health J. 2005;11(4):618-624.

36. Horowitz JA, Damato EG, Duffy ME, Solon L. The relationship of maternal attributes, resources, and perceptions of postpartum experiences to depression. Res Nurs Health. 2005;28(2):159-171. doi:10.1002/nur.20068

37. Klein T, Eckhard J. Educational differences, value of children and fertility outcomes in Germany. Curr Sociol. 2007;55(4):505-525. doi:10.1177/0011392107077636

38. Kahn RS. Depression and unintended pregnancy in young women. Paper raises at least three concerns. BMJ. 2002;324(7345):1097.

39. Rajabi M, Hasheminia F. Children's Value and Fertility Behavior in Shiraz. Iranian Society of Demography. 2013;8 (15):23-42. 